\title{
ESTUDO DE CASOS SOBRE TRANSFERÊNCIA DE TECNOLOGIA PARA SPIN-OFFS UNIVERSITÁRIOS EM PORTUGAL
}

\author{
Vasco Eiriz \\ PhD em Management the University of Manchester Institute of Science and Technology, Reino Unido \\ Professor Associado com Agregação, Departamento de Gestão, Escola de Economia e Gestão, \\ Universidade do Minho, Portugal \\ E-mail: veiriz@eeg.uminho.pt (Portugal)
}

\section{Liliana Alves}

Mestre em Marketing e Gestão Estratégica, Escola de Economia e Gestão, Universidade do Minho, Portugal

E-mail: lilianalousinha@gmail.com (Portugal)

\section{Ana Paula Faria}

$\mathrm{PhD}$ em Economia the University of Nottingham, Reino Unido

Professora Auxiliar com Agregação, Departamento de Economia, Escola de Economia e Gestão, Universidade do Minho, Portugal

E-mail: apfaria@eeg.uminho.pt (Portugal)

\section{RESUMO}

A universidade é uma fonte para o desenvolvimento de novos produtos e serviços baseados no conhecimento e tecnologia. A transferência de tecnologia da universidade para a indústria pode ser feita através de vários mecanismos como a criação de spin-offs universitários. Este artigo analisa quatro spin-offs universitários e os gabinetes de apoio à transferência de tecnologia e empreendedorismo de duas universidades portuguesas, contribuindo, desta forma, para um melhor conhecimento sobre um fenómeno emergente em vários países. Os resultados encontrados revelam que a universidade se mostra mais presente na fase inicial do processo de transferência. A entrada de financiadores viabiliza os spin-offs, mas na maioria dos casos implica para o universitário fundador a perda da maioria do capital e do controlo do spin-off criado em contexto académico. No processo de transferência de tecnologia para os spin-offs universitários identificaram-se ainda as seguintes principais dificuldades: custos associados à inovação, défice de investimento, e conquista de credibilidade e de parceiros estratégicos.

Palavras-chave: Tecnologia; Spin-off; Universidade; Transferência. 


\section{INTRODUÇÃO}

Tecnologia, spin-off universitário e empreendedorismo académico são fenómenos que têm ganho importância no meio académico e fora dele. Para Bercovitz e Feldmann (2006) e Ndonzuau, Pirnay e Surlemont (2002), Silicon Valley e Route 128 são a prova dos primeiros passos do empreendedorismo académico nos Estados Unidos da América. Apesar de em muitos países se encontrar ainda num estado menos desenvolvido, o spin-off académico tem vindo a alterar a cultura de ensino e investigação universitária desde a década de 1970 (Rothaermel, Agung, \& Jiang, 2007).

A possibilidade de comercializar os resultados de pesquisas académicas reforçou a ligação da universidade à indústria e à sua região (Etzkowitz, Webster, Gebhardt, \& Terra, 2000; Gras, Lapera, Solves, Jover, \& Azur, 2008). Esta comercialização do conhecimento conheceu duas vagas. A primeira, na década de 1980, diz respeito à criação de parques de ciência com o objectivo de atrair empresas de vanguarda e aumentar a colaboração com a indústria, contribuindo para o financiamento da pesquisa universitária. Apesar de as iniciativas da primeira vaga ainda serem actuais, surgiu uma segunda vaga na década de 1990 que se focou nos spin-offs e no processo de patentear e licenciar tecnologias. Esta vaga proporcionou aos investigadores um maior envolvimento na comercialização de conhecimento, trabalhando mais de perto com a pressão que implica a transferência de conhecimento e desenvolvimento de produtos com valor económico (Rasmussen, 2006).

A criação de spin-offs universitários tem por base a transferência de tecnologia ou conhecimento da universidade para a nova empresa, constituída, na maioria das vezes, por professores, alunos e investigadores. Quando a transferência tem base tecnológica, esta pode estar ou não protegida legalmente através de patentes. A transferência de tecnologias sob a forma de patentes é um processo complexo. A multiplicidade de factores que condiciona este processo de transferência de tecnologia torna-o com especial interesse de estudo. Este artigo contribui para a compreensão do processo de transferência de tecnologia através do estudo de quatro spin-offs gerados em duas universidades e dos gabinetes que apoiam esse processo no seio das respectivas universidades.

O objectivo deste artigo é, assim, compreender o processo de transferência de tecnologia para spin-offs universitários. Em particular, pretendeu-se: perceber quais as funções que os gabinetes universitários de apoio à transferência de tecnologia e empreendedorismo desempenham no processo; compreender o processo de transferência da tecnologia para spin-offs; identificar mecanismos de ligação do spin-off à universidade; perceber o papel do financiamento no processo de transferência e desenvolvimento do spin-off; e identificar as principais dificuldades associadas ao processo. Para atingir estes objectivos, o método de pesquisa mais adequado é o estudo de caso. Foram estudados quatro spin-offs universitários e três gabinetes universitários de transferência de tecnologia

Revista de Administração e Inovação, São Paulo, v. 9, n. 1, p.167-187, jan./mar. 2012. 
pertencentes à Universidade do Minho e à Universidade do Porto, duas das principais universidades portuguesas.

O presente artigo está organizado da seguinte forma: a secção 2 apresenta a revisão de literatura; a secção 3 descreve a abordagem metodológica seguida na pesquisa empírica; a secção 4 apresenta e analisa os resultados; e, por último, a secção 5 sintetiza os resultados e apresenta as conclusões.

\section{REVISÃO DA LITERATURA}

\subsection{SPIN-OFF COMO MECANISMO DE TRANSFERÊNCIA}

A ligação da academia ao exterior desenrola-se através de diferentes mecanismos de transferência de conhecimento. Os autores que investigaram estes mecanismos identificaram a criação de projectos de pesquisa e laboratórios financiados, as práticas de consultoria, o apoio industrial para a ciência, colaborando e gerindo em conjunto projectos de pesquisa, o patentear, o envolvimento comercial directo em parcerias com empresas, e a contratação de estudantes (Bercovitz \& Feldmann, 2006; Louis, Blumenthal, Gluck, \& Stoto, 1989; Martinelli, Meyer, \& Von Tunzelmann, 2008).

Contudo, a transferência de conhecimento e tecnologia não pode ser dissociada do seu acto criativo e do seu autor. Thursby e Thursby (2003) concluíram que mais de metade das invenções licenciadas pelas universidades americanas não podem ser comercializadas sem a cooperação dos membros universitários.

De acordo com Howells (1995), dois tipos de conhecimento são normalmente transferidos: o conhecimento codificado e o conhecimento tácito. O conhecimento codificado é a parte mais visível dos resultados de pesquisas, podendo assumir diversas formas tais como publicações, programas de computador, artefactos técnicos ou equipamentos (Pirnay, Surlemont, \& Nlemvo, 2003). No entanto, o conhecimento codificado é apenas um conhecimento formal e explícito que representa a ponta do iceberg de outro conhecimento mais profundo e de difícil transmissão: o conhecimento tácito (Fontes, 2005; Goldfarb \& Henrekson, 2003; Gorman, 2002; Howells, 1995; Link \& Scott, 2005; Pirnay et al., 2003).

O indivíduo é o recipiente deste conhecimento tácito e transferi-lo para o exterior por vezes só é possível com um contacto próximo e de grande frequência. A sabedoria, a experiência e as capacidades que o investigador adquire ao longo da sua vida académica fazem parte das formas que o conhecimento tácito assume. É ele que está na origem da tomada de uma decisão, em julgamentos, na organização da informação, na ideia de um produto, e que acaba por não se resumir a uma fórmula 
(Gorman, 2002; Pirnay et al., 2003). Neste sentido vários autores defendem o contacto directo entre o inventor e o processo de desenvolvimento da ideia, pois existe um conhecimento tácito implícito que não pode ser ignorado, dando ao criador um papel decisor no sucesso da aplicação da sua ideia na indústria e mercado (Gartner, 1988; Shane, 2003).

A constituição de uma nova empresa para transferir conhecimento e tecnologia universitária é o mecanismo que envolve maior ligação entre o inventor, a tecnologia transferida e o mercado em que esta é aplicada (Bercovitz \& Feldmann, 2006). A literatura focada em aspectos específicos do processo de criação e desenvolvimento do spin-off apresenta definições deste conceito que variam em certos pontos, mas que acabam por referir-se ao mesmo fenómeno (Bercovitz \& Feldmann, 2006; Pirnay et al., 2003). Empresas formadas pela universidade, professores ou equipas académicas; empresas formadas em torno de uma licença universitária de propriedade intelectual; empresas start-up que desenvolvem projectos de pesquisa conjunta com a universidade; empresa iniciada por estudantes ou pós-doutorados em torno de uma pesquisa conduzida na universidade (Bercovitz \& Feldmann, 2006) são algumas das definições encontradas.

Para Pirnay et al. (2003), o spin-off pode ser definido como sendo uma nova empresa criada para explorar comercialmente algum conhecimento, tecnologia ou resultado de pesquisa desenvolvida com a universidade. Os autores identificaram três condições para que determinado fenómeno possa ser qualificado como spin-off: ter lugar numa organização existente, geralmente apelidada de organização mãe; um ou vários indivíduos possuírem um estatuto ou função na organização mãe; os indivíduos fundadores deixam a organização mãe para criar uma nova empresa.

Clarysse e Moray (2004) argumentaram que o spin-off baseado em pesquisa possui duas dimensões comuns, sendo uma nova empresa que é formada (1) por um membro do corpo docente, da equipa universitária ou por um estudante que partiu da universidade para fundar a empresa ou começou a empresa enquanto estava ainda filiado com a universidade, e/ou (2) possui uma base tecnológica (ou ideia) que é transferida da organização mãe. De acordo com a definição destes autores, o spin-off pode ser visto como um mecanismo de transferência tecnológica para a comercialização da tecnologia desenvolvida na universidade. As novas empresas baseadas em tecnologia são um exemplo do processo de transferência tecnológica da universidade para o mercado (Aguirre, Parellada, \& Campos, 2006; Kirwan, Sijde, \& Groen, 2006).

Vohora, Wright e Lockett (2004) defendem que o spin-off académico passa por um número distinto de fases durante o seu desenvolvimento. Este processo é caracterizado por interactividade e pouca linearidade, onde a constante tomada de decisões e superação de obstáculos é necessária. Durante a progressão entre fases, os autores identificaram quatro conjunturas críticas intrínsecas às 
fases de desenvolvimento do spin-off, relacionando-se estas com capacidades e recursos da nova empresa. As fases de desenvolvimento propostas são: (1) fase de pesquisa; (2) fase de enquadramento de oportunidade; (3) fase de pré-organização; (4) estado de reorganização, e por último, (5) fase de retorno sustentável. As quatro conjunturas críticas são: (A) o reconhecimento da oportunidade; (B) o compromisso empresarial; (C) a credibilidade e (D) os retornos sustentáveis. Assim, o reconhecimento da oportunidade (A) surge como interface entre a fase de pesquisa e a fase de enquadramento da oportunidade. E assim sucessivamente.

Embora o spin-off seja um mecanismo de transferência de conhecimento e tecnologia, o seu sucesso e a forma como é gerido esse processo de transferência dependem largamente das políticas de cada universidade e da ligação do spin-off à universidade.

\subsection{POLÍTICAS UNIVERSITÁRIAS E LIGAÇÃO DO SPIN-OFF À UNIVERSIDADE}

A relação entre o académico e a universidade pode influenciar a forma como o académico age dentro do seu meio, bem como a sua motivação para empreender (Brennan, Wall, \& McGowan, 2005). A organização mãe desempenha um papel crucial no incentivo e apoio do spin-off. Di Gregorio e Shane (2003) concluíram que as razões pelas quais algumas universidades geram mais start-ups do que outras são a eminência intelectual dos membros da universidade e as políticas universitárias praticadas no sentido de apoiar acções empreendedoras. Tais conclusões justificam o facto de algumas universidades apresentarem um impacto muito reduzido na economia regional, com fraco índice de aplicação dos benefícios das pesquisas em spin-offs (Feldman \& Desrochers, 2003, 2004).

Assim, para que a universidade possua meios de transferência de conhecimento, não se pode restringir a um quadro docente altamente qualificado. São necessárias medidas que tornem a transferência viável. Deste modo, as políticas universitárias são uma das variáveis mais influentes no processo de transferência tecnológica (Bercovitz \& Feldmann, 2006; DeGroof \& Roberts, 2004). Neste sentido, uma das evidências encontradas foi a de que a acção das políticas universitárias em torno da transferência tecnológica melhora os serviços de licenciamento tecnológico. O baixo valor de royalties e a propensão para fazer investimento de capital são as medidas com maior interferência neste tipo de actividades (Di Gregorio \& Shane, 2003).

Num estudo realizado no Reino Unido (Wright, Binks, Lockett, \& Vohora, 2003) foram identificados os seguintes promotores da actividade de criação de spin-offs: incentivos e recompensas para os académicos; competências técnicas, de negociação e em marketing que a equipa envolvida na exploração da propriedade intelectual possui; e processos internos relacionados com direitos de propriedade intelectual, constituição de novas empresas e condução e desenvolvimento de negócios. Gras et al. (2008) indicam o baixo valor de royalties, apoios financeiros, serviços de consultoria e Revista de Administração e Inovação, São Paulo, v. 9, n. 1, p.167-187, jan./mar. 2012. 
formação, infraestruturas de apoio como parques tecnológicos e incubadoras como sendo as medidas que mais influência têm no sucesso dos spin-offs e do empreendedorismo académico, em geral.

De acordo com Shane (2004), a política de propriedade intelectual praticada pela universidade é um factor preponderante na geração deste tipo de empresa. Nos Estados Unidos da América a lei Bayh-Dole Act permitiu que as invenções realizadas dentro do seio académico passassem a ter a assinatura da instituição académica em vez de do inventor. Na Europa, os estudos apontam as políticas nacionais que permitem a assinatura individual da invenção como inibidoras da atitude empresarial dos docentes e da actividade spin-off em geral (Goldfarb \& Henrekson, 2003; Wallmark, 1997).

O envolvimento directo da universidade com as actividades de patentear e licenciar e a criação de gabinetes de transferência de tecnologia mostraram-se um processo de sucesso (Sampat, 2006; Shane, 2004). Acrescentam-se os acordos com a indústria e linhas de acção no sentido de grande suporte e selectividade na promoção do spin-off como políticas de promoção da qualidade e garantia de sustentabilidade da nova empresa (DeGroof \& Roberts, 2004). O grau de autonomia concedido aos centros de transferência de tecnologia assim como a todos os que utilizam licenças em troca de capital é o factor mais positivamente relacionado com a formação de novos empreendimentos. Tal sugere que as políticas da universidade e estratégias relativas à estrutura dos centros de transferência e estratégias desenvolvidas para valorizar a propriedade intelectual podem determinar os seus resultados relativos à actividade do spin-off (Gras et al., 2008; Markman, Phan, Balkin, \& Gianiodis, 2005).

Todavia, se por um lado alguns autores como Clarysse, Wright, Lockett, Van de Velde e Vohora (2005) e Di Gregorio e Shane (2003) defendem que um elevado grau de envolvimento entre a universidade e o spin-off pode trazer benefícios tais como maiores probabilidades de sobrevivência, melhor desempenho e efeitos positivos na sua reputação; por outro lado, Johansson, Jacob e Hellstrom (2005) e Rothaermel e Thursby (2005) argumentam que o elevado envolvimento pode causar situações de dependência, danos na reputação e atraso no desenvolvimento.

Johansson et al. (2005) distinguem os laços fortes dos laços fracos entre a nova empresa e a universidade. Os laços fortes tendem a ser bidireccionais e informais, ajudando diariamente o spin-off, tornando o projecto viável. Por sua vez, os laços fracos tendem a ser unidireccionais e formais, contribuindo para o acesso a aspectos importantes para a nova empresa. As relações históricas profissionais levam a que o académico desenvolva laços fortes com a universidade onde trabalha. A proximidade geográfica, a confiança e o espírito de cooperação entre os membros do seu departamento, assim como a partilha da mesma linguagem e conhecimento são as causas de os spinoffs académicos serem apoiados por um pequeno número de laços fortes desenvolvidos em torno da universidade. 
Peréz e Sánchez (2003) constataram que a organização mãe tem um papel muito importante nas primeiras etapas da nova empresa, auxiliando no desenvolvimento do produto, prestando serviços de consultoria e ajudando a atingir alguma experiência. No entanto, apesar deste papel preponderante da organização mãe, a tendência é a de que com o passar dos anos a ligação à universidade diminua, aumentando as relações com os clientes. Rothaermel e Thursby (2005) defendem que as ligações com a universidade asseguram um crescimento mais estável mas que retardam o amadurecimento da nova empresa.

\section{ESTUDO DE CASOS}

O método de investigação utilizado foi o estudo de caso. De acordo com Yin (2003), o estudo de caso é uma investigação empírica que estuda um fenómeno dentro do seu contexto real, sendo desenvolvido especialmente quando as fronteiras entre o contexto e o fenómeno em estudo não são claras. Desta forma, procurou-se através do estudo comparativo entre casos acrescentar algo de novo ao tema, ainda que, naturalmente, os resultados alcançados não possam ser generalizados.

Os casos foram escolhidos por razões de conveniência e interesse dos investigadores. Por exemplo, a proximidade geográfica das duas universidades foi considerada na medida em que isso favoreceu o contacto com as instituições no decorrer da investigação. A escolha recaiu sobre a Universidade do Minho e a Universidade do Porto. Esta escolha teve também em consideração a existência de gabinetes com o objectivo de incentivar e apoiar a transferência de conhecimento e criação de spin-offs nas duas universidades. Apesar de os autores deste estudo estarem vinculados a uma das universidades estudadas, nenhum deles exerce funções nos gabinetes e nos spin-offs estudados, nem sequer têm responsabilidades institucionais na área, tendo sido garantida a imparcialidade e independência entre autores e objecto de estudo. Aliás, esta ligação a uma das universidades permitiu-lhes uma leitura mais informada do fenómeno.

O estudo desenrolou-se em torno do processo de transferência de tecnologia sob a forma de patentes. Esta transferência de conhecimento tecnológico teria, por sua vez, de ser feita para spin-offs universitários, procurando assim perceber os mecanismos que melhor contribuem para a transferência de tecnologia da universidade. A pesquisa deste fenómeno num determinado contexto corrobora o facto de o estudo de caso ser o método mais apropriado para o efeito. Yin (1994) caracteriza as questões de partida do estudo de caso como sendo de ordem exploratória, onde o como e o porquê estão habitualmente presentes.

A principal questão de pesquisa a que nos propusemos responder foi a seguinte: como se desenrola o processo de transferência de tecnologia, sob a forma de patentes, para um spin-off 
universitário? De forma mais particular, qual é o papel dos gabinetes de transferência neste processo? Que mecanismos de ligação existem entre as universidades e os spin-offs aí gerados? Que papel exerce a necessidade de financiamento no processo de transferência, criação e desenvolvimento dos spin-offs? Quais são as dificuldades associadas ao processo de transferência através de spin-offs?

Em consequência da principal questão exposta, torna-se importante esclarecer o conceito de spin-off universitário adoptado no nosso estudo. Como a revisão de literatura evidenciou, o número de definições de spin-off universitário é grande, sendo que muitos autores referem-se ao mesmo fenómeno em termos diferentes (Bercovitz e Feldmann, 2006; Pirnay et al., 2003). A definição utilizada neste estudo é a de Clarysse e Moray (2004), que apresentam o spin-off como uma nova empresa que é formada por um membro do corpo docente da universidade ou por um estudante que partiu da universidade para fundar a empresa ou começou a empresa enquanto estava ainda filiado à universidade, e possui uma base tecnológica (ou ideia) que é transferida da universidade enquanto organização mãe.

A selecção dos spin-offs estudados teve em conta outro critério: a tecnologia transferida devia estar patenteada. A existência de uma patente pressupõe a protecção dos direitos industriais sobre determinado conhecimento técnico, o que fez com que a escolha dos spin-offs tenha incidido em empresas tecnológicas nascentes em diferentes áreas da engenharia. $\mathrm{Na}$ escolha dos casos, a transferência devia já ter sido concretizada, tendo o spin-off no mínimo três anos de existência, uma vez que consideramos que um spin-off em fase muito precoce de formação não apresenta uma experiência nem uma visão retrospectiva sobre o processo de transferência e desenvolvimento da empresa tão amadurecida como outros spin-offs em fases mais avançadas.

Foram estudados quatro spin-offs, dois da Universidade do Minho (Ambisys e Micropolis) e dois da Universidade do Porto (Medmat Innovation e Fluidinova). O Quadro 1 caracteriza esses spinoffs. 


\begin{tabular}{|c|c|c|c|c|}
\hline \multirow{2}{*}{$\begin{array}{l}\text { Características dos } \\
\text { Spin-offs }\end{array}$} & \multicolumn{2}{|c|}{ Universidade do Minho } & \multicolumn{2}{|c|}{ Universidade do Porto } \\
\hline & Ambisys & Micropolis & $\begin{array}{c}\text { Medmat } \\
\text { Innovation }\end{array}$ & Fluidinova \\
\hline Área & $\begin{array}{l}\text { Biotecnologia } \\
\text { ambiental }\end{array}$ & $\begin{array}{l}\text { Engenharia } \\
\text { têxtil }\end{array}$ & Biomateriais & Engenharia química \\
\hline Início da investigação & 1999 & 1998 & 1989 & Não disponível \\
\hline N. ${ }^{\circ}$ investigadores & 5 & 2 & 3 & "Vários investigadores" \\
\hline $\begin{array}{l}\text { Ligação fundador- } \\
\text { universidade }\end{array}$ & $\begin{array}{l}\text { Professora e } \\
\text { aluno de } \\
\text { doutoramento }\end{array}$ & $\begin{array}{l}\text { Professor e } \\
\text { aluno de } \\
\text { doutoramento }\end{array}$ & $\begin{array}{l}\text { Professores } \\
\text { investigadores }\end{array}$ & $\begin{array}{l}\text { Professores } \\
\text { investigadores }\end{array}$ \\
\hline $\begin{array}{l}\text { N. }{ }^{\circ} \text { de pedidos de } \\
\text { patentes }\end{array}$ & 2 & 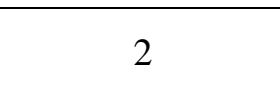 & 3 & 6 \\
\hline Fundação do spin-off & 2007 & 2001 & 2002 & 2005 \\
\hline $\begin{array}{l}\mathrm{N}{ }^{\circ} \text { de investigadores } \\
\text { empreendedores }\end{array}$ & 2 & 2 & 1 & Não disponível \\
\hline $\begin{array}{l}\text { N. }{ }^{\circ} \text { de patentes } \\
\text { exploradas }\end{array}$ & 1 & 1 & 3 & 2 \\
\hline $\begin{array}{l}\text { Histórico de } \\
\text { financiamento }\end{array}$ & $\begin{array}{l}\text { Grupo privado } \\
\text { (2007) }\end{array}$ & $\begin{array}{l}\text { Capital de risco } \\
\text { (2003) e } \\
\text { business angel } \\
\text { (2007) }\end{array}$ & $\begin{array}{l}\text { Grupo privado } \\
\text { (2006) }\end{array}$ & Capital de risco (2005) \\
\hline $\begin{array}{l}\text { Localização actual em } \\
\text { Portugal }\end{array}$ & $\begin{array}{c}\text { Póvoa de } \\
\text { Varzim }\end{array}$ & TecMaia Maia & TecMaia Maia & TecMaia Maia \\
\hline $\begin{array}{l}\text { Estado de } \\
\text { desenvolvimento do } \\
\text { spin-off }\end{array}$ & $\begin{array}{c}\text { Experimentação } \\
\text { em empresas }\end{array}$ & Comercialização & Comercialização & $\begin{array}{c}\text { Comercialização de } \\
\text { amostras }\end{array}$ \\
\hline $\begin{array}{l}\text { Outras actividades do } \\
\text { spin-off }\end{array}$ & $\begin{array}{l}\text { Prestação de } \\
\text { serviços e } \\
\text { consultoria }\end{array}$ & Não aplicável & Biomodelação & Consultoria \\
\hline
\end{tabular}

Quadro 1 - Características dos spin-offs estudados.

Fonte: Elaborado pelos autores.

A recolha de dados baseou-se em três fontes: a consulta dos sítios na internet das universidades, dos spin-offs universitários e dos gabinetes universitários de apoio à transferência de tecnologia e ao empreendedorismo; a análise de documentos dos gabinetes universitários; e as entrevistas realizadas a estes últimos e aos professores fundadores.

As duas primeiras fontes foram utilizadas para caracterizar de forma preliminar as empresas e os gabinetes de transferência, e para preparar as entrevistas. Foram posteriormente realizadas quatro entrevistas a professores que estiveram envolvidos em cada uma das tecnologias transferidas, e que viveram o processo de protecção e transferência da patente para o spin-off, bem como a criação deste. Para além destas entrevistas, foram realizadas três entrevistas nos gabinetes de transferência das universidades.

A duração média destas sete entrevistas foi de 55 minutos. Todas as entrevistas foram gravadas em áudio. A realização das entrevistas foi apoiada por um guião com os seguintes tópicos de 
conversação: i) enquadramento do gabinete de transferência e seu papel na universidade; ii) processo de transferência, incluindo fases, motivos e dificuldades; iii) constituição do spin-off; iv) ligação entre o spin-off e a universidade; e v) financiamento do spin-off. Naturalmente, este guião foi adaptado em função de o entrevistado ser um professor ou um técnico do gabinete de transferência.

O tratamento de dados seguiu o método proposto por Miles e Huberman (1994). A primeira fase do tratamento dos dados das entrevistas consistiu na sua audição e transcrição integral para um documento MS Word. De seguida, os tópicos abordados nas entrevistas foram categorizados em tabelas nas quais foram estruturados os dados através de excertos das entrevistas e registo de ideiaschave que traduziram a nossa análise e interpretação desses dados. Conseguiu-se desta forma uma simplificação dos dados recolhidos, tornando a análise e comparação dos dados, bem assim como a interpretação dos resultados, mais fácil e evidente.

Os resultados são apresentados e discutidos na seguinte secção.

\section{APRESENTAÇÃO E DISCUSSÃO DE RESULTADOS}

\subsection{FUNÇÃO DOS GABINETES DE TRANSFERÊNCIA}

A origem universitária da patente confere particularidades ao processo interno de comunicação de invenção na universidade, anterior ao pedido legal de patente. A Universidade do Minho e a Universidade do Porto possuem gabinetes de apoio à transferência de tecnologia que auxiliam os investigadores a patentear as tecnologias.

A TecMinho é uma associação que presta apoio à Universidade do Minho, enquanto a Universidade do Porto Inovação (UPIN) é o gabinete de apoio da Universidade do Porto, sendo a TecMinho uma associação de direito privado e a segunda um departamento da reitoria. No caso da Universidade do Porto, destaca-se ainda a existência de gabinetes internos de apoio nesta área dentro de algumas faculdades, como é o exemplo dos Serviços de Cooperação da Faculdade de Engenharia da Universidade do Porto. Isto ocorre porque a Universidade do Porto é uma instituição bem maior e mais antiga do que a Universidade do Minho e com uma tradição de autonomia das suas faculdades mais enraizada. A Universidade do Porto foi fundada em 1911, possuindo aproximadamente 29 mil estudantes e 2.300 professores e investigadores distribuídos por 15 faculdades e 69 centros de investigação. A Universidade do Minho foi fundada em 1973, estando localizada nas cidades de Braga e Guimarães, com uma população estudantil de aproximadamente 16 mil alunos e 1.200 professores e investigadores repartidos por 11 faculdades e 35 centros de investigação.

Revista de Administração e Inovação, São Paulo, v. 9, n. 1, p.167-187, jan./mar. 2012. 
Apesar de a reitoria de cada universidade ser a decisora no processo de pedido de patente, são os gabinetes mencionados que elaboram pareceres sobre o pedido de patente, organizam formação para os potenciais empreendedores e dão o apoio necessário no processo de pedido de patente e sua transferência.

Sem prejuízo do reconhecimento da autoria da invenção, a titularidade da universidade sobre a invenção pressupõe que o autor desta comunique ao reitor ou entidade por ele nomeada, no caso da Universidade do Minho, ou à UPIN, no caso da Universidade do Porto, a sua invenção ou criação. A decisão de patentear a invenção ou criação é, em ambos os casos, da responsabilidade da universidade.

A Universidade do Minho por norma emite um parecer fundamentado sobre o seu posicionamento acerca da solicitação de patente, podendo recorrer para o efeito a uma terceira entidade. Na Universidade do Porto cabe à UPIN elaborar um parecer fundamentado acerca da solicitação da patente, que é entregue ao reitor ou outrem por ele designado, sendo o reitor, com o auxílio das assessorias que considere oportunas, quem decide sobre o interesse da universidade de solicitar a patente.

Quando a decisão é positiva, as despesas de protecção são nas duas universidades estudadas suportadas por estas. A Universidade do Minho paga estas despesas através dos seus serviços centrais, enquanto no Regulamento de Propriedade Intelectual da Universidade do Porto está estabelecido o dever de a universidade suportar as despesas de protecção sem que seja mencionado nenhum serviço ou organismo interno específico para este pagamento. No decorrer do processo de patentear a inovação são a TecMinho e a UPIN que trabalham junto com os inventores, pois, apesar de a escolha dos países onde o direito sobre a patente vai vigorar ser dos autores, os gabinetes aconselham os autores neste processo.

No que diz respeito à exploração da patente, a Universidade do Minho e a Universidade do Porto possuem a competência de decidir sobre a exploração dos direitos de propriedade industrial e o poder de praticar todos os actos que levem à exploração da mesma, de acordo com os seus regulamentos de propriedade intelectual. Similarmente, nas duas universidades, o inventor ou o inventor e a sua faculdade, no caso da Universidade do Porto, têm por sua vez o direito de serem informados sobre o processo decorrente da exploração dos direitos de propriedade da patente.

A exploração da patente é feita através da transferência da patente para uma empresa via licenciamento exclusivo. Este licenciamento tem como contrapartida o pagamento de royalties à universidade licenciadora, passando a empresa exploradora da patente a pagar os custos da sua protecção nos países em que o direito de propriedade industrial da inovação vigora. 
Quando os autores da patente decidem acompanhar esta até ao mercado e criam um spin-off que tem por base a patente da qual são autores, a Universidade do Minho e a Universidade do Porto não financiam as novas empresas, sendo o apoio que prestam a nível de serviços e da disponibilização de espaços incubadores. Nestes casos, a TecMinho e a UPIN auxiliam através do encaminhamento para entidades financiadoras, organização de acções de formação e indicação de parceiros que podem prestar apoio no desenvolvimento do plano de negócios. Neste sentido, os gabinetes universitários têm um papel de intermediários entre o fundador do spin-off e potenciais parceiros deste. $\mathrm{O}$ acesso a redes de contactos importantes é assim uma das funções desempenhadas pelos gabinetes.

\subsection{LIGAÇÃO DOS SPIN-OFFS À UNIVERSIDADE}

Para além do auxílio anteriormente identificado, a universidade pode também conceder a sua marca ao spin-off, sendo que a Universidade do Minho possui a marca Spin-off da Universidade do Minho, enquanto a Universidade do Porto concede a chancela Spin-off U. Porto. O estatuto de spinoff da Universidade do Minho é concedido quando o spin-off tem uma ligação com a universidade. A concessão da chancela Spin-off U. Porto está sujeita a critérios estabelecidos no regulamento específico para a concessão da chancela. Ao contrário do que acontece na Universidade do Minho, na Universidade do Porto a chancela Spin-off U. Porto está sujeita a um regulamento próprio, não dependendo apenas da filiação dos fundadores à universidade.

$\mathrm{Na}$ Universidade do Porto, a concessão da chancela passa ainda por um processo administrativo que envolve a análise e elaboração de um parecer pela UPIN e uma comissão de acompanhamento que delibera sobre o parecer de atribuir ou não a chancela. A concessão da marca mostra-se mais fácil na Universidade do Minho, pois, enquanto nesta universidade existiam no momento da recolha de dados 34 spin-offs com o estatuto anteriormente referido, na Universidade do Porto existia apenas um spinoff com a chancela Spin-off U. Porto, apesar de outros spin-offs usufruírem do apoio e laços com a Universidade do Porto. Nas duas universidades, o uso da marca é por tempo indefinido, não existindo um limite temporal estabelecido para o spin-off deixar de poder usar a marca da universidade.

A ligação à universidade mostrou-se mais evidente na fase inicial do spin-off através dos gabinetes de apoio à transferência de tecnologia e empreendedorismo da universidade. Este vínculo tornou-se importante na rede de contactos da nova empresa. No caso da Ambisys, esta estabelece a sua rede de contactos através da universidade, grupo financiador e via comercial. A Micropolis, por seu lado, desenvolveu a sua rede de contactos através dos contactos pessoais do professor e dos contactos da empresa de capital de risco, tendo também sido estabelecidos contactos através da universidade.

Revista de Administração e Inovação, São Paulo, v. 9, n. 1, p.167-187, jan./mar. 2012. 
O papel da universidade no desenvolvimento da rede de contactos foi também referido pela Medmat Innovation e pela Fluidinova. A Medmat Innovation criou a sua rede via universidade, via contactos pessoais dos professores fundadores, desenvolvendo a rede com o crescimento da empresa e a aquisição de novos contactos através dos já existentes. O mesmo acontece na Fluidinova, onde a rede de contactos foi constituída via universidade e via projectos de participação do spin-off.

\subsection{PROCESSO DE TRANSFERÊNCIA PARA OS SPIN-OFFS}

Os quatro casos de transferência analisados neste estudo estão ligados à engenharia, tendo envolvida a transferência de duas ou mais patentes em cada caso. Conforme pode ser visto no Quadro 1, entre os quatro spin-offs estudados existe um na área da biotecnologia ambiental (Ambisys), outro na engenharia têxtil (Micropolis), um terceiro na engenharia de biomateriais (Medmat Innovation) e o quarto na engenharia química (Fluidinova).

A invenção que deu origem à patente resulta em todos os casos de uma investigação universitária de professores com alunos de doutoramento dentro da sua área de formação. O número de pessoas envolvidas na investigação é entre dois e cinco, não existindo informação neste item no caso da Fluidinova.

A Ambisys demorou oito anos entre o início da investigação e a sua fundação. A Micropolis, por sua vez, demorou três anos entre o início da investigação e a fundação da empresa, enquanto a Medmat Innovation é aquela que apresenta uma distância temporal maior, sendo esta de treze anos.

É importante realçar o facto de a criação dos spin-offs ter ocorrido em diferentes fases da aplicação da tecnologia. Enquanto a formação da Ambisys e da Micropolis ocorreu anos antes da comercialização da tecnologia, no caso da Medmat Innovation esta foi criada quando a tecnologia estava praticamente pronta para ser comercializada.

A constituição da equipa fundadora do spin-off é em todos os casos caracterizada por uma ligação dos fundadores à universidade através da docência e da investigação. Realça-se o facto de os quatro professores fundadores entrevistados se situarem numa faixa etária entre 46 e 59 anos, sendo de admitir que a estabilidade na carreira profissional e a maturidade de conhecimentos no momento da criação dos spin-offs possam ter contribuído para a decisão empreendedora.

A decisão de transferir a tecnologia para spin-offs em vez de a desenvolver e investir nela no contexto exclusivo da universidade foi um tópico analisado neste estudo. Quando interrogados sobre o motivo de transferir a patente, os entrevistados deram respostas que convergiram em dois grandes motivos: ser uma tecnologia inovadora e ter potencial de aplicação.

Revista de Administração e Inovação, São Paulo, v. 9, n. 1, p.167-187, jan./mar. 2012. 
A Ambisys argumentou que a tecnologia era bastante revolucionária e com grande potencial de aplicação no mercado global. A Micropolis referiu que a tecnologia desenvolvida implementa um novo conceito de conforto térmico. No caso da Medmat Innovation, apesar de directamente não ter referido a inovação e o potencial de aplicação da tecnologia, mencionou a importância da tecnologia na área médica a que se dirige. A Fluidinova apontou a possibilidade de aplicação prática da tecnologia e o seu grande potencial como factores de transferência dessa mesma tecnologia.

Associados aos motivos de transferência da tecnologia - inovação e potencial de mercado existiram outras causas particulares para que a criação do spin-off fosse considerada o melhor modo para transferir a patente. Assim, os motivos para a criação do spin-off variam de caso para caso. A Ambisys e a Micropolis são opostos neste item, pois, enquanto a primeira resultou de um impulso externo, no caso da Micropolis a sua criação resultou da motivação de um dos autores da patente.

No caso da Ambisys a criação do spin-off resultou de um convite feito por um grupo empresarial que não se encontrava no mercado da biotecnologia ambiental, mas tinha como objectivo entrar neste mercado. Através de uma ligação entre a professora, uma das autoras da patente, e uma antiga aluna que trabalhava no grupo empresarial estabeleceu-se o contacto e o encontro de interesses do grupo e de alguns autores da patente em querer investir na tecnologia. De forma oposta, na Micropolis a decisão da criação do spin-off resultou da motivação pessoal de um dos autores da patente, resultante da sua vontade de ligar o seu conhecimento académico à indústria têxtil. A esta motivação pessoal aliou-se o conhecimento inovador da patente, sendo que a tecnologia implícita na patente não tinha concorrência no mercado.

No caso da Medmat Innovation, o sector médico para o qual a patente tem aplicação prática possui regulamentos específicos para certificar uma nova tecnologia, levando um dos autores da primeira patente a criar o spin-off para desenvolver a patente e resolver os aspectos regulamentares necessários para a sua aplicação no mercado. Relativamente à Fluidinova foi a relevância da invenção conjugada com a constatação de um interesse externo na tecnologia e do seu potencial valor comercial que levaram à criação do spin-off.

\subsection{FINANCIAMENTO DOS SPIN-OFFS E DIFICULDADES NA TRANSFERÊNCIA}

A criação de uma nova empresa acarreta consigo necessidades financeiras relacionadas com o estabelecimento e seu crescimento. O financiamento dos spin-offs estudados foi realizado através de grupos privados (Ambisys e Medmat Innovation) e de sociedades de capitais de risco (Micropolis e Fluidinova). Analisando a distribuição de poder dentro dos spin-offs estudados a conclusão é evidente:

Revista de Administração e Inovação, São Paulo, v. 9, n. 1, p.167-187, jan./mar. 2012. 
com a entrada de um financiador externo, em três dos casos estudados a maioria do capital passou a pertencer ao financiador, enquanto os fundadores ficaram minoritários.

Por exemplo, na Ambisys, o grupo empresarial tem $70 \%$ da empresa, enquanto os fundadores possuem $10 \%$ cada. O poder do grupo e o esforço dos restantes três sócios é reconhecido pela professora entrevistada, que admite a possibilidade de futuramente terem que reduzir a sua posição accionista em virtude de os três sócios não conseguirem acompanhar os aumentos de capital realizados pelo grupo empresarial.

O poder maioritário do financiador é também evidente na Micropolis, sendo também uma sociedade anónima de capital de risco que ficou com a maioria do capital do spin-off. Tendo os fundadores cerca de $10 \%$, o poder de decisão ficou controlado pela sociedade de capital de risco. Por seu lado, a Medmat Innovation, que integrou o grupo Biosckin criado pelos fundadores do spin-off e o grupo empresarial investidor, é exemplo de um poder de decisão mais repartido, embora a maioria accionista seja também do grupo empresarial investidor.

Neste aspecto, a Fluidinova assemelha-se aos restantes, tendo a sociedade de capital de risco a maioria accionista, enquanto os promotores possuem apenas $20 \%$, existindo ainda uma posição de $15 \%$ que pertence ao Estado. Realça-se ainda o facto de o cargo que os professores fundadores ocupam na empresa ser o de administrador e director científico/tecnológico.

O financiamento externo traz consigo vantagens e condicionalismos, tendo sido enumerados alguns deles. A Ambisys reconheceu as seguintes vantagens: acesso facilitado ao mercado; a estrutura sólida do grupo, já com imagem externa de grande credibilidade; e facilidade de financiamento bancário. A valorização do conhecimento académico e a predisposição para investir e tornar viável o desenvolvimento da tecnologia e do spin-off foram outras vantagens reconhecidas pela Medmat Innovation e indirectamente pela Fluidinova.

Relacionados com os condicionalismos do financiamento foram mencionados pela Ambisys a necessidade de respeitar a política do grupo financiador que condiciona as grandes decisões e estratégia do próprio spin-off. De forma comum com a Micropolis, foi mencionada também pela Ambisys a dificuldade em acompanhar o financiador nos aumentos de capital. A Micropolis acrescentou também a dificuldade de conseguir obter da sociedade de capital de risco todo o investimento que precisava, sendo que, a cada investimento efectuado, era exigida como contrapartida um produto final mais rapidamente.

A Micropolis apontou a divergência de opiniões quanto ao tempo de espera pelo produto final como um ponto muito sensível no relacionamento entre investigador e investidor, ansiando o 
investidor pelo retorno imediato do financiamento, apesar de todo o tempo necessário ao apuramento de uma nova tecnologia.

Para além das dificuldades de financiamento existem outras dificuldades associadas ao processo de transferência de patente para um spin-off universitário. A Ambisys enunciou défice de investimento na área ambiental; a conquista da confiança e credibilidade no mercado; o encontro de parceiros adequados e honrados; e os problemas de adaptação de uma tecnologia inovadora criada em laboratório ao mercado como sendo as principais dificuldades sentidas na transferência da tecnologia patenteada para o spin-off e para o mercado. Por sua vez, a Micropolis referiu as seguintes dificuldades: tempo associado à produção de produtos novos e sofisticados; dificuldades de logística e estruturas necessárias à comercialização; os preços praticados; os pré-requisitos de qualidade do produto e de produção; o clima pouco favorável à inovação na indústria têxtil; e a localização geográfica do spin-off.

A Medmat Innovation identificou a forte regulamentação na área médica, a escassa formação nas áreas regulamentares em dispositivos médicos, a insuficiência de financiamentos e a ausência de apoios da parte das faculdades e universidades como as maiores dificuldades sentidas no processo de transferência da tecnologia para o spin-off. Por fim, a Fluidinova realçou o facto de a tecnologia ser para um mercado organizacional. O tempo de apuramento da tecnologia, os custos e riscos associados ao conhecimento inovador, a conquista de espaço no mercado, bem como a própria ausência de formação dos professores empreendedores em gestão foram outros aspectos importantes referidos pelo professor entrevistado e responsável pela Fluidinova.

\section{CONCLUSÃO}

Nas duas universidades estudadas foi clara a presença de regulamentos de propriedade intelectual e de gabinetes de apoio à transferência de tecnologia e empreendedorismo académico. No que diz respeito aos regulamentos, as duas universidades actuam de forma similar sobre os direitos de autor e titularidade da invenção, sendo o autor da invenção reconhecido como tal, mas sendo a titularidade invenção da universidade onde foi gerada.

A transferência de tecnologia realizada na Universidade do Minho e na Universidade do Porto é feita por norma através de licenciamento exclusivo, ficando o spin-off obrigado a suportar os custos relacionados com a protecção da invenção, pagando ainda à universidade royalties estabelecidos previamente.

A universidade mostrou-se mais presente numa fase inicial através do apoio prestado pela TecMinho e pela UPIN na submissão do pedido de patente. Realça-se o facto de a Universidade do Revista de Administração e Inovação, São Paulo, v. 9, n. 1, p.167-187, jan./mar. 2012. 
Porto possuir gabinetes específicos em algumas faculdades para apoio à transferência de tecnologia e ao empreendedorismo, podendo assim prestar um apoio mais directo e próximo do que o gabinete de apoio geral da universidade.

Por outro lado, verificou-se que as universidades contribuem para a afirmação dos spin-offs através da concessão da sua marca, tendo sido referido pelas quatro empresas estudadas como algo muito positivo no estabelecimento de redes de contactos e da imagem pública. A Universidade do Minho concede a marca Spin-off da Universidade do Minho, enquanto a Universidade do Porto concede a chancela Spin-off U. Porto. Apesar de a marca poder ser usada por um período ilimitado nas duas universidades, os critérios de concessão da marca parecem ser bem mais restritos no caso da Universidade do Porto, pois, enquanto esta possui apenas um spin-off com a chancela Spin-off $\mathbf{U}$. Porto, a Universidade do Minho tem 34 spin-offs com estatuto definido.

Apesar das dificuldades sentidas na transferência de tecnologia e criação de spin-offs, os professores fundadores das quatro empresas estudadas identificaram razões semelhantes para acompanhar a transferência da patente da qual eram autores. Assim, as respostas convergiram em dois motivos fundamentais: a tecnologia ser inovadora e possuir potencial de aplicação.

Contudo, o facto de a transferência de tecnologia ser feita para uma nova empresa teve em cada caso motivações específicas. No caso dos spin-offs da Universidade do Minho as motivações são opostas. Num caso foi a motivação pessoal da parte do professor, no outro caso, o impulso foi externo uma vez que resultou do convite de um grupo privado que tencionava entrar na área da tecnologia. Por sua vez, nos casos da Universidade do Porto as motivações para criar os spin-offs estiveram relacionadas num dos casos com questões de regulação da área a que a tecnologia pertencia, enquanto no outro caso foi o próprio potencial da tecnologia e o interesse externo que impulsionaram a criação da nova empresa.

No que diz respeito ao financiamento, nenhuma das duas universidades financia os seus spinoffs, tendo os gabinetes universitários de apoio apenas a função de informar sobre possíveis financiadores e fazer o respectivo encaminhamento. Dois spin-offs, um da Universidade do Minho e outro da Universidade do Porto, foram financiados por grupos privados com interesse nos mercados onde as tecnologias seriam aplicadas. Por seu lado, os outros dois spin-offs foram financiados através de sociedades de capital de risco. Contudo, a entrada de financiadores externos implicou uma nova distribuição de poder entre professores fundadores e financiadores, ficando em três dos quatro casos estudados o fundador com uma percentagem minoritária do capital.

As afinidades entre financiadores e fundadores não foram em todos os casos as mais evidentes, pois divergências de opiniões sobre o rumo do spin-off, natureza de conhecimentos distintos e vários 
tipos de pressões foram alguns dos aspectos referidos como críticos nessa relação. Contudo, nos quatro casos estudados foi reconhecida a importância do financiamento como viabilizador da existência do spin-off e como contribuindo para construção de espaços próprios de incubação, uma vez que nenhum spin-off estudado incubou na própria universidade.

As principais dificuldades do processo de transferência identificadas neste estudo foram as seguintes: tempo de desenvolvimento e lançamento do produto no mercado; custos associados à inovação e protecção da tecnologia; detecção de parceiros; construção de credibilidade junto de parceiros; dificuldades associadas ao conhecimento inovador e à sua adaptação à escala necessária para comercialização das tecnologias; dificuldades logísticas; dificuldades de financiamento; clima pouco favorável à inovação; e regulamentação e pré-requisitos de qualidade do produto e da produção.

Através deste estudo ficamos a compreender melhor o processo de transferência de conhecimento sob a forma de tecnologias patenteadas. Verificámos, em particular, qual o papel que os gabinetes de transferência de duas universidades exercem no processo, que mecanismos de ligação se estabelecem entre os spin-offs e as suas universidades de origem, e as dificuldades sentidas no processo. Nesta medida, o estudo aqui apresentado, embora tenha permitido ilustrar de forma relativamente completa cada um dos quatro casos estudados, possui um carácter exploratório que merece ser desenvolvido através de estudos complementares, não só de outros casos de spin-offs (tanto no domínio tecnológico como noutras áreas de conhecimento), mas também através de estudos quantitativos envolvendo populações alargadas de spin-offs de várias universidades de diferentes países.

\section{REFERÊNCIAS}

Aguirre, I. P., Parellada, F. S., \& Campos, H. M. (2006). University spin-off programmes: how can they support the NTBF creation?. International Entrepreneurship and Management Journal, 2(2), 157-172.

Bercovitz, J., \& Feldmann, M. (2006). Entrepreneurial universities and technology transfer: a conceptual framework for understanding knowledge-based economic development. Journal of Technology Transfer, 31(1), 175-188.

Brennan, M. C., Wall, A. P., \& McGowan, P. (2005). Academic entrepreneurship: assessing preferences in nascent entrepreneurs. Journal of Small Business and Enterprise Development, 12(3), 307-322.

Clarysse, B., \& Moray, N. (2004). A process study of entrepreneurial team formation: the case of a research-based spin-off. Journal of Business Venturing, 19(1), 55-79.

Revista de Administração e Inovação, São Paulo, v. 9, n. 1, p.167-187, jan./mar. 2012. 
Clarysse, B., Wright, M., Lockett, A., Van de Velde, E., \& Vohora, A. (2005). Spinning out new ventures: a typology of incubation strategies from European research institutions. Journal of Business Venturing, 20(2), 183-216.

Degroof, J.-J., \& Roberts, E. B. (2004). Overcoming weak entrepreneurial infrastructures for academic spin-off ventures. Journal of Technology Transfer, 29(3/4), 327-352.

Di Gregorio, D., \& Shane, S. (2003). Why do some universities generate more start-ups than others?. Research Policy, 32(2), 209-227.

Etzkowitz, H., Webster, A., Gebhardt, C., \& Terra, B. R. C. (2000). The future of the university and the university of the future: evolution of ivory tower to entrepreneurial paradigm. Research Policy, 29(2), 313-330.

Feldman, M. P., \& Desrochers, P. (2004). Truth for its own sake: academic culture and technology transfer at the Johns Hopkins University. Minerva, 42(2), 105-126.

Feldman, M. P., \& Desrochers, P. (2003). Research universities and local economic development: lessons from the history of Johns Hopkins University. Industry and Innovation, 10(1), 5-24.

Fontes, M. (2005). The process of transformation of scientific and technological knowledge into economic value conducted by biotechnology spin-offs. Technovation, 25(4), 339-347.

Gartner, W. B. (1988). Who is an entrepreneur? Is the wrong question. American Journal of Small Business, 12(4), 47-68.

Goldfarb, B., \& Henrekson, M. (2003). Bottom-up versus top-down policies towards the commercialization of university intellectual property. Research Policy, 32(4), 639-658.

Gorman, M. E. (2002). Types of knowledge and their roles in technology transfer. Journal of Technology Transfer, 27(3), 219-231.

Gras, J. M. G., Lapera, D. R. G., Solves, I. M., Jover, A. J. V., \& Azur, J. S. (2008). An empirical approach to the organizational determinants of spin-off creation in European universities. International Entrepreneurship and Management Journal, 4(2), 187-198.

Howells, J. (1995). Tacit knowledge and technology transfer. Technology Analysis \& Strategic Management, 8(2), 91-106.

Johansson, M., Jacob, M., \& Hellstrom, T. (2005). The strength of strong ties: university spin-offs and the significance of historical relations. Journal of Technology Transfer, 30(3), 271-286.

Kirwan, P., Sijde, P., \& Groen, A. (2006). Assessing the needs of new technology based firms (NTBFs): an investigation among spin-off companies from six European universities. International Entrepreneurship and Management Journal, 2(2), 173-187.

Link, A. N., \& Scott, J. T. (2005). Opening the ivory towers door: an analysis of the determinants of the formation of U.S. university spin-off companies. Research Policy, 34(7), 1106-1112.

Louis, K. S., Blumenthal, D., Gluck, M. E., \& Stoto, M. A. (1989). Entrepreneurs in academe: an exploration of life scientists. Administrative Science Quarterly, 34(1), 110-131. 
Markman, G. D., Phan, P. H., Balkin, D. B., \& Gianiodis, P. T. (2005). Entrepreneurship and university based technology transfer. Journal of Business Venturing, 20(2), 241-263.

Martinelli, A., Meyer, M., \& Von Tunzelmann, N. (2008). Becoming an entrepreneurial university? A case study of knowledge exchange relationships and faculty attitudes in a medium-sized, researchoriented university. Journal of Technology Transfer, 33(3), 259-283.

Miles, M. B., \& Huberman, A. M. (1994). Qualitative data analysis - an expanded sourcebook $\left(2^{\text {nd }}\right.$ ed.). Thousand Oaks: SAGE Publications.

Ndonzuau, F. N., Pirnay, F., \& Surlemont, B. (2002). A stage model of academic spin-off creation. Technovation, 22(5), 281-289.

Pérez, M. P., \& Sánchez, A. M. (2003). The development of university spin-offs: early dynamics of technology transfer and networking. Technovation, 23(10), 823-831.

Pirnay, F., Surlemont, B., \& Nlemvo, F. (2003). Toward a typology of university spin-offs. Small Business Economics, 21(4), 355-369.

Rasmussen, E. (2006). Academic entrepreneurship: a process view of university spin-off venture formation. Recuperado em 31 de agosto, 2009, de http://www.durham-ent.org.

Rothaermel, F. T., Agung, S. D., \& Jiang, L. (2007). University entrepreneurship: a taxonomy of the literature. Industrial and Corporate Change, 16(4), 691-791.

Rothaermel, F. T., \& Thursby, M. C. (2005). Incubator firm failure or graduation? The role of university linkages. Research Policy, 34(7), 1076-1090.

Sampat, B. N. (2006). Patenting and US academic research in the 20th century: the world before and after Bayh-Dole. Research Policy, 35(6), 772-789.

Shane, S. (2004). Encouraging university entrepreneurship? The effect of the Bayh-Dole act on university patenting in the United States. Journal of Business Venturing, 19(1), 127-151.

Shane, S. (2003). A general theory of entrepreneurship: the individual-opportunity nexus. Cheltenham: Edward Elgar.

Thursby, J. G., \& Thursby, M. C. (2003). Are faculty critical? Their role in university-industry licensing (Working Paper n. 9991). Cambridge: NBER.

Vohora, A., Wright, M., \& Lockett, A. (2004). Critical junctures in the development of university high-tech spinout companies. Research Policy, 33(1), 147-174.

Wallmark, J. T. (1997). Inventions and patents at universities: the case of Chalmers University of Technology. Technovation, 17(3), 127-139.

Wright, M., Binks, M., Lockett, A., \& Vohora, A. (2003). Survey on university commercialization activities (Financial Year 2002). Nottingham: NUBS.

Yin, R. K. (2003). Case study research: design and methods ( $3^{\text {rd }}$ ed.). Thousand Oaks: SAGE Publications. 
Yin, R. K. (1994). Case study research: design and methods ( $2^{\text {nd }}$ ed.). Thousand Oaks: SAGE Publications.

\title{
CASE STUDIES ON TRANSFER OF TECHNOLOGY TO UNIVERSITY SPIN-OFFS IN PORTUGAL
}

\begin{abstract}
The university is a source for the development of new products and services based on knowledge and technology. The technology transfer from university to industry can be done through several mechanisms such as the creation of university spin-offs. This article analyzes four spin-offs and the offices that support the technology transfer and entrepreneurship of two Portuguese universities. In turn, this contributes to a better understanding of an emergent phenomenon in several countries. The results show that the university is more active in the early stages of the transfer process. Funding enables the development of the spin-off but in most cases implies that the academic founders lose the majority of capital and the control of their spin-offs launched in the academic context. In the process of technology transfer to the university spin-offs we have identified the following difficulties: costs associated with innovation, investment deficit, achieving credibility and acquiring strategic partners.
\end{abstract}

Key-words: Technology; Spin-off; University; Transfer.

Data do recebimento do artigo: 24/05/2011

Data do aceite de publicação: 18/09/2011

Revista de Administração e Inovação, São Paulo, v. 9, n. 1, p.167-187, jan./mar. 2012. 